Universitätsklinik für Viszerale Chirurgie und Medizin

Viszeral- und Transplantationschirurgie

Dr. med. Andreas Kohler, Prof. Dr. med. Guido Beldi

\title{
Operation bei asymptomatischer Inguinalhernie - unnötig?
}

\section{Zusammenfassung}

Bei Patienten mit asymptomatischer Inguinalhernie wurde bisher zur Prophylaxe von Inkarzerationen grosszügig die Indikation zur Operation gestellt. Die Inkarzeration einer Inguinalhernie ist jedoch ein seltenes und bei rechtzeitigem Eingreifen gut behandelbares Krankheitsbild. Randomisierte Studien haben gezeigt, dass ein abwartendes, primär nicht operatives Vorgehen („Watchful Waiting“) möglich ist und Inkarzerationen die Ausnahme sind. Die Studien haben aber auch gezeigt, dass 50 bis $70 \%$ der Patienten mit asymptomatischer Inguinalhernie im Verlauf von sieben Jahren wegen neu aufgetretenen Symptomen operationspflichtig werden. Eine abwartende Haltung bezüglich Operation ist sicher, jedoch muss der Patient über die Möglichkeit einer Zunahme der Beschwerden mit Notwendigkeit zur operativen Sanierung aufgeklärt werden.

\section{Summary}

Until recently, the repair of asymptomatic groin hernia has been advocated as a prophylaxis for incarceration. However, incarcerations of inguinal hernias are rare events and can be treated adequately if the patient presents himself early after onset of symptoms. Randomized controlled trials showed, that watchful waiting is not associated with increased incarcerations and can be offered as a save alternative. However, within seven years after randomization $50 \%$ to $70 \%$ of the patients in the watchful waiting groups required surgery, mainly because of new onset or increasing symptoms. 


\section{Einleitung}

Die operative Versorgung der Inguinalhernie hat eine Geschichte die bis ins 18. Jahrhundert zurückgeht und ist eine der am häufigsten durchgeführten Operationen überhaupt (1). Zu Beginn nur als Notfalloperation bei inkarzerierten Hernien durchgeführt, hat sich im zwanzigsten Jahrhundert die Technik derart verbessert, dass Komplikationen und Rezidivraten minimiert werden konnten (2). Zunehmend wurde die Indikation zum Hernienrepair bei minimal symptomatischen oder asymptomatischen Patienten gestellt. Die Operation hat bei diesen Patienten im Gegensatz zu den symptomatischen Patienten den Stellenwert einer Prophylaxe zur Vermeidung einer Zunahme der Hernie und vor potenzieller Inkarzeration.

In diesem Artikel wird die Indikationsstellung beim minimal symptomatischen oder asymptomatischen Patienten diskutiert. Es stellt sich die Frage ob ein präventive Operation durchgeführt werden soll, welche potentiell Komplikationen mit sich bringen kann. Dazu muss geklärt werden, wie häufig Inkarzerationen bei abwartendem Verhalten auftreten und bei wie vielen Patienten eine Inguinalhernie im Verlauf der Zeit symptomatisch wird. Grundlage hierfür sind neue randomisierte Studien welche den Verlauf nach Operation mit demjenigen ohne Operation vergleichen. 


\section{Diagnose}

Im Vordergrund steht bei der Diagnosestellung der Inguinalhernie nach wie vor die Anamnese und die klinische Untersuchung. Typischerweise berichtet der Patient über ein Druckgefühl oder eine Schwellung in der Leiste, welche bei erhöhtem intraabdominellem Druck (z.B. Husten, Treppensteigen oder Heben von schweren Lasten) zunehmen. Schmerzen sind für eine Inguinalhernie eher untypisch. Beschwerden welche im Liegen auftreten oder persistieren sprechen für eine andere Ursache.

Die klinische Untersuchung wird im Stehen und Liegen durchgeführt. Im Stehen ist die Hernie besser sichtbar und die maximale Grösse lässt sich genauer abschätzen. Im Liegen lässt sich die Bruchpforte besser untersuchen, da hierbei die Rumpfmuskulatur maximal entspannt ist. Kleine Hernien können durch intraabdominelle Drucksteigerung (Husten oder Heben des Kopfes in Rückenlage) besser identifiziert werden. Beim Mann erfolgt die Palpation indem die skrotale Haut invertiert wird. Die Fingerspitze stülpt hierzu von caudal des Hodens nach cranial lateral zum äusseren Leistenring (siehe Abbildung 1). So wird der distale Leistenkanal durch den äusseren Leistenring ausgetastet, auch hier unter Provokation durch Husten oder Pressen. Differentialdiagnostisch muss insbesondere bei der Frau eine Femoralhernie gesucht werden. In disem Fall verläuft der Bruchsack unterhalb des Leistenbandes entlang der Inguinalgefässe und ist meist deutlich kleiner als bei der direkten oder indirekten Inguinalhernie. Eine (meist diskrete) Schwellung ist bei der Femoralhernie unterhalb des Leistenbandes zu suchen.

Werden trotz Vorliegen der typischen Anamnese bei der körperlichen Untersuchung keine Hinweise für eine Inguinalhernie gefunden, müssen Differentialdiagnosen in Betracht gezogen werden. Häufig sind Leistenschmerzen durch muskuloskelettale Probleme bedingt. Nicht selten manifestiert sich ein Impingement der Hüfte oder eine Arthrose durch inguinale Schmerzen. Auch Zerrungen der Adduktorenmuskulatur sind häufig für Leistenschmerzen verantwortlich. Weitere in Betracht zu ziehende Ursachen sind Lymphadenopathien oder eine Varikozele. Um diese Diagnosen nicht zu verpassen, müssen zusätzlich zu Abdomen und Leiste die Hüfte, die Lymphknoten und das Skrotum untersucht werden.

Wird keine alternative Ursache gefunden, wird zur weiteren Abklärung eine Ultraschalluntersuchung und falls notwendig in einem zweiten Schritt eine diagnostische Laparoskopie durchgeführt. Sollte sich bei der Laparoskopie eine Inguinalhernie zeigen, kann diese in derselben Operation saniert werden. 


\section{Argumente für und gegen die Operation}

\section{Die Inkarzeration - selten}

Die drohende Inkarzeration einer Dünndarmschlinge war über lange Zeit das Hauptargument zur Sanierung einer asymptomatischen Inguinalhernie. Es handelt sich bei der Inkarzeration um eine chirurgische Notfallsituation welche häufig bei älteren Patienten vorkommt und vor 1990 noch mit einer Mortalität von über 10\% einherging (3, 4).

Im Falle einer irreversiblen Schädigung durch die Ischämie ist eine Resektion des inkarzerierten Stücks Darm beziehungsweise Omentum notwendig. Gemäss der Literatur ist eine Darmresektion bei 10-20\% der Patienten mit akuter Inkarzeration notwendig (5-8). Die Operation in der Akutsituation geht mit einer erhöhten Morbidität (verzögerte Passage, Anastomoseninsuffizienz bei Darmresektion, Peritonitis, Sepsis, Kreislaufversagen) einher und wird bei $30-40 \%$ beschrieben. Heutzutage liegt die postoperative Mortalität bei $2-7 \%$ und ist damit verglichen zur elektiven Situation deutlich erhöht (5-8).

Aufgrund fehlender Daten wurde das Risiko für das Auftreten einer Inkarzeration beim Vorliegen einer Inguinalhernie lange Zeit überschätzt. Eine korrekte wissenschaftliche Erfassung das natürlichen Verlaufes einer Inguinalhernie mit Inzidenz und Morbidität einer potentiellen Inkarzeration war aufgrund des seltenen Auftretens einer Inkarzeration und aufgrund der regelhaft durchgeführten Inguinalhernienplastik nicht praktikabel. Seit den Neunzigerjahren wurden erste mutige Studien durchgeführt, welche zeigen, dass die Inkarzeration selten ist. Zwei prospektive Studien untersuchten den natürlichen Verlauf bei Patienten mit Inguinalhernien über knapp zehn Jahre. In der „Watchful Waiting“ Gruppe wurde eine Inzidenz von 2, respektive 3 Inkarzerationen pro 1000 Patientenjahre gefunden. Jedoch muss fesgehalten werden, dass ein Grossteil der Patienten trotz Zuteilung in diese Gruppe im Verlauf elektiv operiert wurde. In den Studienarmen mit Operation traten keine Inkarzerationen auf, es wurden Rezidivraten zwischen 2.5 und 3\% beschrieben. $(9,10)$. Bei diesem sehr geringen Risiko für eine Inkarzeration ist eine Operation, welche ebenfalls mit einer gewissen Morbidität einhergeht, nicht undifferenziert zu empfehlen. 


\section{Früh- und Spätkomplikationen einer elektiven Inguinalhernienplastik - nicht zu unterschätzen}

Wie jede Operation, geht die Inguinalhernienoperation mit spezifisch chirurgischen und allgemeinen Komplikationen einher. Intraoperative Komplikationen wie die Verletzung der im Operationsgebiet liegenden Gefässe mit relevanten Blutungen oder eine Verletzung des Ductus deferens oder der Hodengefässe mit nachfolgender Orchitis oder Hodenatrophie sind möglich. Bei Patienten mit abdominellen Voroperationen ist bei den laparoskopischen Verfahren die Verletzung von Dünndarm oder Blase möglich, was aber selten eintritt. Die Inzidenz dieser intraoperativen Komplikationen liegt bei elektiven Operationen deutlich unter einem Prozent $(11,12)$. Ein postoperativer Harnverhalt tritt in 1-2\% der operierten Patienten auf. Wundinfekte wurden bei ungefähr $1 \%$ der operierten Patienten beschrieben $(11,12)$.

Zu den allgemeinen Komplikationen gehören unter anderem anästhesiologische Probleme, deren Auftreten vor allem von der Schwere der Begleiterkrankungen des Patienten abhängt. Weitere seltene allgemeinen Komplikationen sind die Lungenembolie und die Pneumonie.

Die im klinischen Alltag relevanteste Komplikation ist der chronische postoperative Leistenschmerz. Diese Schmerzen können für den Patienten eine deutliche Einschränkung darstellen und sind oft schwer zu therapieren. Die Angaben bezüglich Inzidenz chronischer postoperativer Schmerzen nach Hernienoperation hängen sehr von der Definition dieser Schmerzen ab. Ein Jahr nach Inguinalhernienrepair sind nur $60 \%$ der Patienten vollständig asymptmatisch. Zu diesem Zeitpunkt leiden 9-11\% der Patienten an relevanten Schmerzen mit einem Wert von über 3 auf der Visual Analog Skala (VAS 0-10) (13-15). Es wurde gezeigt, dass diese Schmerzen seltener nach laparoskopischem Hernienrepair auftreten (16).

Die nicht zu unterschätzende Inzidenz dieser Komplikationen, macht eine ausgewogene Indikationsstellung und eingehende Aufklärung unumgänglich. 


\section{Natürlicher Verlauf der asymptomatischen Inguinalhernie - häufig kommt es trotzdem zur Operation}

Im Jahre 2006 wurden zwei randomisierte Studien durchgeführt, welche ein „Watchful Waitig“ gegenüber einer primären operativen Versorgung bei männlichen Patienten verglichen $(9,10)$. In der Gruppe der nicht operierten Patienten wurden 17\%, respektive $19 \%$ der Patienten wegen zunehmenden Beschwerden innerhalb von einem Jahr trotzdem elektiv operiert. Auch über die folgenden Jahre zeigte sich weiterhin eine zunehmende Anzahl Patienten aus der Beobachtungsgruppe welche sich elektiv operieren liessen, so dass nach sieben Jahren 50-70\% der Patienten in den Beobachtungsgruppen operiert waren (siehe Abbildung 2). Basierend auf diesen Daten kann aufgrund der tiefen Inkarzerationsraten weiterhin davon ausgegangen werden, dass Abwarten ein sicheres Vorgehen ist. Jedoch muss dem Patienten beim Entscheid zu einem „Watchful Waiting“ bewusst sein, dass eine Operation wegen zunehmenden Beschwerden im Verlauf nicht unwahrscheinlich ist. Dies wiederum rechtfertigt auch den Entscheid zu einer sofortigen Operation, falls dies vom Patienten gewünscht ist.

Prognostische Faktoren bezüglich Progredienz von Symptomatik und Grösse der Hernie wurden nur am Rande untersucht. Es bleibt unklar, ob die Grösse der Hernie bei der initialen Vorstellung, das Alter oder die körperliche Aktivität des Patienten einen Einfluss auf den Wunsch bezüglich operativer Sanierung im Verlauf haben. Weiter wäre es interessant zu wissen, ob eine Korrelation zwischen Grössenzunahme der Hernie über die Zeit und Operationswunsch des Patienten besteht. Mit diesen Informationen könnten Patientengruppen identifiziert werden, bei welchen eine abwartende Strategie besonders sinnvoll ist.

\section{Schlechte Compliance des Patienten - problematisch bei abwartendem Verhalten}

Betrachtet man die Patientengruppe, welche eine notfallmässige Operation bei inkarzerierter Inguinalhernie benötigt, fält ein deutlicher Zusammenhang zwischen der Zeitspanne von Symptombeginn bis zur Operation und dem Outcome auf. Es konnte gezeigt werden, dass bei Patienten welche innerhalb von 24h nach Symptombeginn operiert wurden, signifikant weniger Darmresektionen nötig waren als bei Patienten welche erst über 24h Stunden nach den initialen Symptomen der Inkarzeration operiert wurden (17).

Die Erfahrungen aus der Praxis bestätigen diese Tatsache. Indolente Patienten und diejenigen welche einen Arztbesuch herauszögern, präsentieren sich öfter in einem 
späten Stadium der Inkarzeration, welches bereits mit einer Nekrose des inkarzerierten Darms einhergeht. Diese Situation erfordert eine Resektion, was wiederum mit einer höheren Morbidität und Mortalität einhergeht.

\section{Operationstechnik}

Für die Sanierung einer Inguinalhernie stehen heute die Techniken mit einer Netzeinlage im Vordergrund. Die Operation kann entweder offen (meist Technik nach Lichtenstein) oder laparoskopisch durchgeführt werden. Bei der offenen Technik kommt das Netz direkt vor der Transversalisfaszie zu liegen, bei den laparoskopischen Techniken in einer präperitonealen Schicht.

Ein Vorteil der Operation nach Lichtenstein ist die Durchführbarkeit in Lokal- oder Spinalanästhesie, wodurch bei polymorbiden Patienten, mit grossem Risiko für eine Allgemeinanästhesie, diese umgangen werden kann. Beim Vorliegen eines untypischen Tastbefundes in der Leiste kann über einen Zugang nach Lichtenstein die Leiste exploriert und danach gemäss Befund die Operation weitergeführt werden.

Studien welche laparoskopische mit offenen Verfahren vergleichen, zeigen eine Tendenz zur schnelleren postoperativen Erholung sowie zu weniger postoperativen chronischen Schmerzen bei der Laparoskopie (16). Ein Vorteil der laparoskopischen Techniken liegt darin, dass beide Seiten über denselben Zugang saniert werden können. Besteht auf der einen Seite eine symptomatische Hernie, auf der anderen eine asymptomatische, soll hier die Indikation zur beidseitigen Versorgung grosszügig gestellt werden. Damit kann mit minimalem zusätzlichem Aufwand eine Zweitoperation vermieden werden. Weiter hat man durch den laparoskopischen Zugang die Möglichkeit den Bauchraum und die Leistenregion von innen zu inspizieren, was insbesondere bei unklaren Situationen sehr hilfreich sein kann. 


\section{Entscheidungsfindung bei asymptomatischer Inguinalhernie}

Spricht die Anamnese und der erhobene Untersuchungsbefund für das Vorliegen einer Hernie sind keine weiteren Abklärungen nötig. Die operative Sanierung der Hernie ist in diesem Fall indiziert (siehe Abbildung 3).

Die aktuell verfügbare Literatur zeigt, dass beim Vorliegen einer Inguinalhernie welche asymptomatisch oder lediglich minimal symptomatisch ist, die Operationsindikation differenziert gestellt werden kann. Beim Patienten welcher wegen fehlenden Symptomen keine Operation wünscht, ist eine zuwartende Strategie gerechtfertigt, da das Risiko einer Inkarzeration als gering einzustufen ist. Diese Patienten müssen aber darauf aufmerksam gemacht werden, dass Beschwerden durch die Hernie häufig über die Zeit auftreten und dann eine Sanierung der Hernie indiziert ist. Weil der Anteil der Patienten welche im Verlauf wegen zunehmenden Beschwerden eine Operation benötigen hoch ist, kann eine Inguinalhernienplastik auch dem asymptomatischen Patienten empfohlen werden. In Zukunft sind weitere Studien notwendig, mit dem Ziel Patienten mit hohem Risiko für eine Progredienz der Hernie besser zu identifizieren.

In der aktuellen Literatur sind weibliche Patienten mit Inguinalhernie und alle Patienten mit Femoralhernie ausgeschlossen. Die Inzidenz von Femoralhernien ist deutlich tiefer, jedoch treten bei Femoralhernien relativ mehr Inkarzerationen und damit Notfallsituationen mit komplikationsträchtigem Verlauf auf $(8,18)$. Bei Frauen ist der Anteil an Femoralhernien deutlich höher als bei Männern (19). Ein „Watchful Waiting“ ist bei Femoralhernien wissenschaftlich nicht untersucht und scheint aufgrund der vorliegenden Fakten auch nicht sinnvoll.

Entscheidet sich ein Patient mit asymptomatischer Inguinalhernie für eine „Watchful Waiting" Strategie, muss er instruiert werden, sich bei plötzlich auftretenden Schmerzen im Bereich der Hernie oder bei lleuszeichen notfallmässig ärztlich vorzustellen, damit Abklärungen bezüglich einer Inkarzeration sofort gemacht werden können. Bei Patienten mit geringer Compliance oder bei Patienten welche sich öfters in Ländern mit eingeschränktem Gesundheitssystem aufhalten ist eher zur primären Operation zu raten, da in diesen Situationen eine adäquate Versorgung bei einer Inkarzeration nicht zeitgerecht durchgeführt werden könnte. In diesen Fällen ist eine gute Kommunikation zwischen Patient, Hausarzt und Operateur wichtig.

Bei laparoskopisch durchgeführten Hernienoperationen kann bei intraoperativer Diagnose einer asymptomatischen Inguinalhernie auf der Gegenseite auch eine bilaterale 
Inguinalhernienplastik durchgeführt werden. Der Patient soll über diese Situation präoperativ aufgeklärt werden und sein Einverständnis geben.

Zusammenfassend ist eine asymptomatische Inguinalhernie keine zwingende Operationsindikation. Ein abwartendes, konservatives Vorgehen kann dem Patienten als Alternative zur Operation vorgeschlagen werden, mit dem Hinweis, dass die Hernie im Verlauf von Monaten bis Jahren mit grosser Wahrscheinlichkeit symptomatisch wird und dann operiert werden muss.

\section{Literaturverzeichnis}

1. Sanders DL, Kingsnorth AN. From ancient to contemporary times: a concise history of incisional hernia repair. Hernia : the journal of hernias and abdominal wall surgery. 2012;16(1):1-7.

2. Matthews RD, Anthony T, Kim LT, Wang J, Fitzgibbons RJ, Jr., Giobbie-Hurder A, et al. Factors associated with postoperative complications and hernia recurrence for patients undergoing inguinal hernia repair: a report from the VA Cooperative Hernia Study Group. American journal of surgery. 2007;194(5):611-7.

3. Lewis DC, Moran CG, Vellacott KD. Inguinal hernia repair in the elderly. Journal of the Royal College of Surgeons of Edinburgh. 1989;34(2):101-3.

4. Tingwald GR, Cooperman M. Inguinal and femoral hernia repair in geriatric patients. Surgery, gynecology \& obstetrics. 1982;154(5):704-6.

5. Kulah B, Duzgun AP, Moran M, Kulacoglu IH, Ozmen MM, Coskun F. Emergency hernia repairs in elderly patients. American journal of surgery. 2001;182(5):455-9.

6. Alvarez JA, Baldonedo RF, Bear IG, Solis JA, Alvarez P, Jorge JI. Incarcerated groin hernias in adults: presentation and outcome. Hernia : the journal of hernias and abdominal wall surgery. 2004;8(2):121-6.

7. Abi-Haidar Y, Sanchez V, Itani KM. Risk factors and outcomes of acute versus elective groin hernia surgery. Journal of the American College of Surgeons. $2011 ; 213(3): 363-9$.

8. Venara A, Hubner M, Le Naoures P, Hamel JF, Hamy A, Demartines N. Surgery for incarcerated hernia: short-term outcome with or without mesh. Langenbeck's archives of surgery / Deutsche Gesellschaft fur Chirurgie. 2014;399(5):571-7.

9. Fitzgibbons RJ, Jr., Giobbie-Hurder A, Gibbs JO, Dunlop DD, Reda DJ, McCarthy $M$, Jr., et al. Watchful waiting vs repair of inguinal hernia in minimally symptomatic men: a randomized clinical trial. JAMA : the journal of the American Medical Association. 2006;295(3):285-92.

10. O'Dwyer PJ, Norrie J, Alani A, Walker A, Duffy F, Horgan P. Observation or operation for patients with an asymptomatic inguinal hernia: a randomized clinical trial. Annals of surgery. $2006 ; 244(2): 167-73$.

11. Lundstrom KJ, Sandblom G, Smedberg S, Nordin P. Risk factors for complications in groin hernia surgery: a national register study. Annals of surgery. 2012;255(4):784-8.

12. El-Dhuwaib Y, Corless D, Emmett C, Deakin M, Slavin J. Laparoscopic versus open repair of inguinal hernia: a longitudinal cohort study. Surgical endoscopy.

2013;27(3):936-45.

13. Palmqvist E, Larsson K, Anell A, Hjalmarsson C. Prospective study of pain, quality of life and the economic impact of open inguinal hernia repair. The British journal of surgery. 2013;100(11):1483-8. 
14. Bay-Nielsen M, Perkins FM, Kehlet $\mathrm{H}$. Pain and functional impairment 1 year after inguinal herniorrhaphy: a nationwide questionnaire study. Annals of surgery.

$2001 ; 233(1): 1-7$.

15. Nienhuijs S, Staal E, Strobbe L, Rosman C, Groenewoud H, Bleichrodt R. Chronic pain after mesh repair of inguinal hernia: a systematic review. American journal of surgery. 2007;194(3):394-400.

16. Bittner R, Schwarz J. Inguinal hernia repair: current surgical techniques.

Langenbeck's archives of surgery / Deutsche Gesellschaft fur Chirurgie. 2012;397(2):271-

82.

17. Koizumi M, Sata N, Kaneda Y, Endo K, Sasanuma H, Sakuma Y, et al. Optimal timeline for emergency surgery in patients with strangulated groin hernias. Hernia : the journal of hernias and abdominal wall surgery. 2014.

18. van den Heuvel B, Dwars BJ, Klassen DR, Bonjer HJ. Is surgical repair of an asymptomatic groin hernia appropriate? A review. Hernia : the journal of hernias and abdominal wall surgery. 2011;15(3):251-9.

19. Burcharth J, Pedersen M, Bisgaard T, Pedersen C, Rosenberg J. Nationwide prevalence of groin hernia repair. PloS one. 2013;8(1):e54367.

20. Schumpelick V. Hernien, 4. Auflage, 2000.

\section{Korrespondenzadresse}

Prof. Dr. G. Beldi

Universitätsklinik für Viszerale Chirurgie und Medizin

Inselspital, 3010 Bern, Schweiz

guido.beldi@insel.ch 
Abbildung 1: Untersuchung des Leistenkanals durch den äusseren Leistenring (Abbildung aus (20))

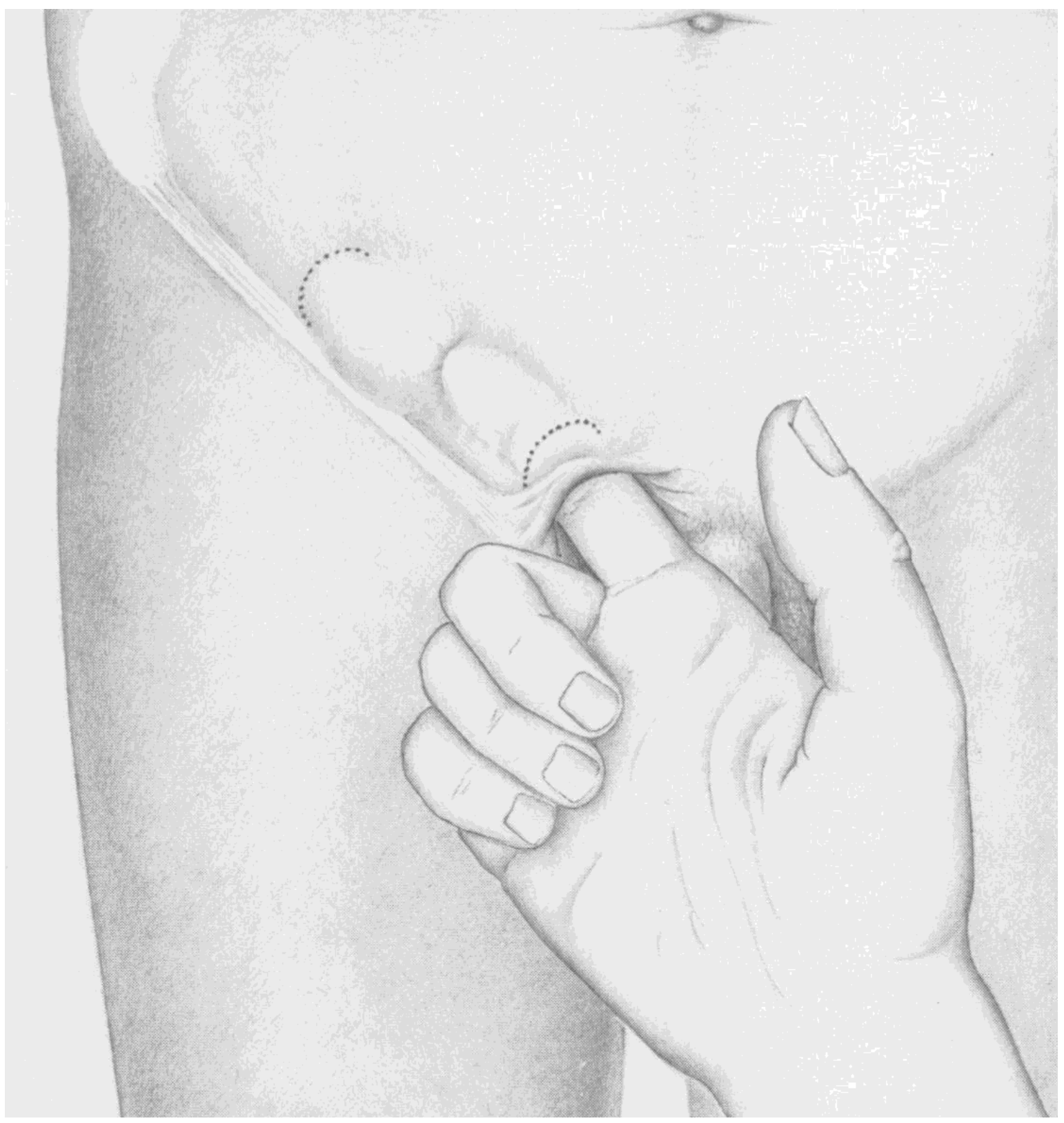


Abbildung 2: Randomisierte kontrollierte Studien welche „Watchful Waiting“ gegen sofortige Operation vergleichen (modifiziert nach $(9,10)$ ): Anteil an Patienten welche über die Zeit wegen zunehmenden Symptomen operiert wurden

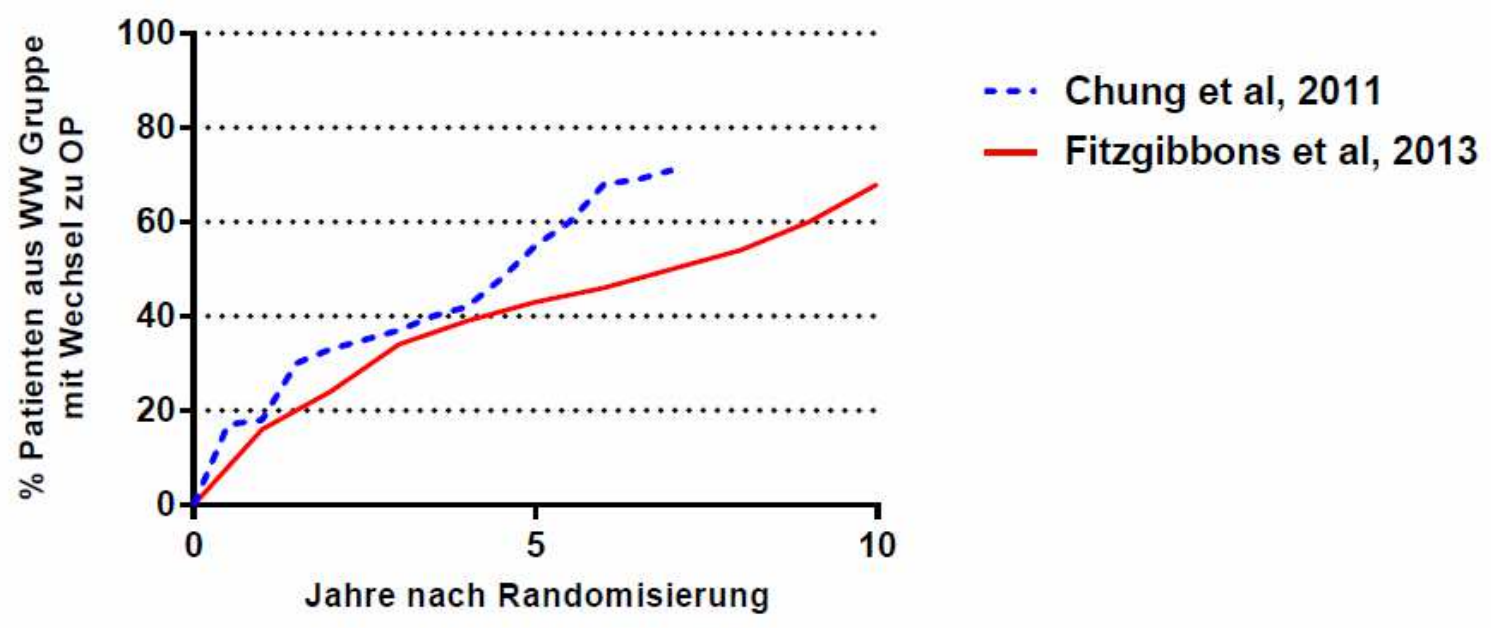


Abbildung 3: Flussdiagramm Indikationsstellung Inguinalhernienrepair

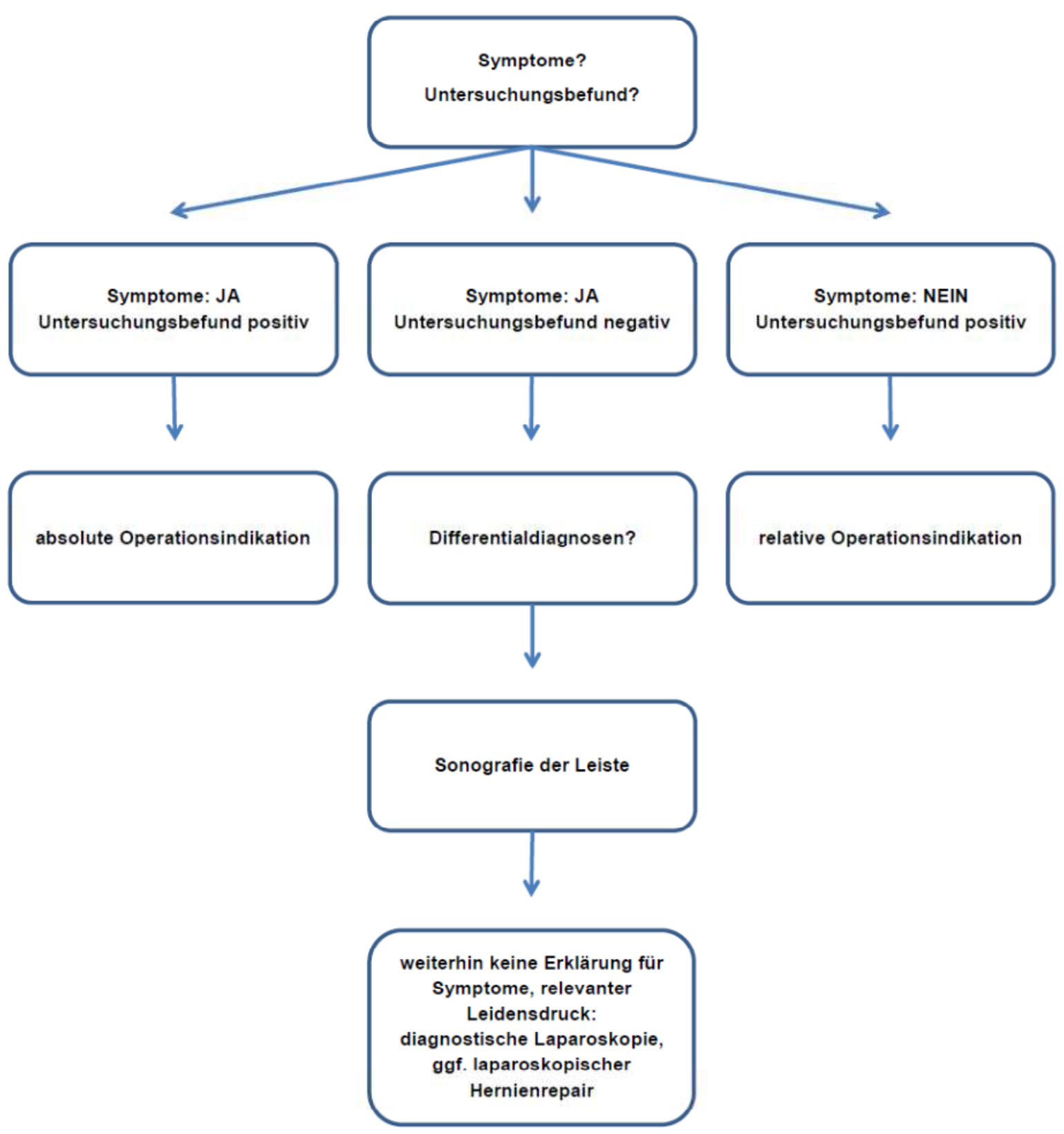

